\title{
A pathway profile-based method for drug repositioning
}

\author{
YE Hao $^{1,2}$, YANG LinLin $^{1,2}$, CAO ZhiWei ${ }^{3}$, TANG KaiLin ${ }^{2 *} \&$ LI YiXue ${ }^{1,2 *}$ \\ ${ }^{1}$ State Key Laboratory of Bioreactor Engineering, East China University of Science \& Technology, Shanghai 200237, China; \\ ${ }^{2}$ Shanghai Center for Bioinformation Technology, Shanghai 200235, China; \\ ${ }^{3}$ School of Life Science and Technology, Tongji University, Shanghai 200092, China
}

Received August 1, 2011; accepted December 6, 2011; published online February 23, 2012

\begin{abstract}
Finding new applications for existing pharmaceuticals, known as drug repositioning, is a validated strategy for resolving the problem of high expenditure but low productivity in drug discovery. Currently, the prevalent computational methods for drug repositioning are focused mainly on the similarity or relevance between known drugs based on their "features", including chemical structure, side effects, gene expression profile, and/or chemical-protein interactome. However, such drug-oriented methods may constrain the newly predicted functions to the pharmacological functional space of the existing drugs. Clinically, many drugs have been found to bind "off-target" (i.e. to receptors other than their primary targets), which can lead to undesirable effects. In this study, which integrates known drug target information, we propose a disease-oriented strategy for evaluating the relationship between drugs and disease based on their pathway profile. The basic hypothesis of this method is that drugs exerting a therapeutic effect may not only directly target the disease-related proteins but also modulate the pathways involved in the pathological process. Upon testing eight of the global best-selling drugs in 2010 (each with more than three targets), the FDA (Food and Drug Administration, USA)-approved therapeutic function of each was included in the top 10 predicted indications. On average, $60 \%$ of predicted results made using our method are proved by literature. This approach could be used to complement existing methods and may provide a new perspective in drug repositioning and side effect evaluation.
\end{abstract}

drug repositioning, pathway profile, pharmacological function, drug-disease relationship

Citation: $\quad$ Ye H, Yang L L, Cao Z W, et al. A pathway profile-based method for drug repositioning. Chin Sci Bull, 2012, 57: 2106-2112, doi: 10.1007/s11434$012-4982-9$

Drug discovery is a time-consuming and laborious process. To bring a single de novo drug to the market, an average of more than $\$ 800$ million is spent in a time period of $\sim 15$ years [1]. Moreover, about $90 \%$ of drugs fail during development in phase I clinical trials [2], a rate caused mainly by findings of low therapeutic efficacy and/or unacceptable toxicity $[3,4]$. Others are discovered to have similar drawbacks, even after approval by drug regulatory authorities (e.g. the US Federal Drug Administration (FDA)), or postmarketing. In exceptional circumstances, it is necessary to either modify the drug or even withdraw it from the market. The "thalidomide affair", a highly recognizable example of a drug having tragic unintended effects, is a typical example. Thalidomide was withdrawn from the market in the early

*Corresponding authors (email: yxli@ @scbit.org; kltang@ scbit.org) 1960s because of teratogenic effects. However, it has since been reborn as an FDA-approved treatment for cancers and lepriasis owing to the discovery of new indications, namely immunomodulatory and anti-angiogenic activities [5-8].

Despite enormous increases in novel technologies over recent times, the productivity of drug discovery efforts has actually decreased since the mid-1990s [9]. The need to define a novel strategy and/or utilize new resources in drug discovery is urgent. Drug repositioning, which aims to discover new indications for existing drugs, can shorten the development cycle and reduce the risk of unacceptable pharmacokinetics and safety profiles. Any newly identified functions can be quickly evaluated in phase II clinical trials [10]. Currently, there are four typical computational methods in drug repositioning: (i) predicting new drug targets on the basis of the chemical structure of the drug together with 
known information regarding its targets [11]; (ii) identifying relationships between drugs based on the similarity of their side effects [12]; (iii) analyzing gene expression following drug treatment to infer new indications [13]; and (iv) building a background chemical-protein interactome (CPI) using molecular docking and existing data describing drugs and adverse reaction proteins [14]. In this final method, the association between a queried small molecule and drugs within the CPI is used to develop potential indications. All of these methods are good predictors of different aspects of drug-like activities, and some have been successfully verified experimentally [11]. However, any newly discovered indications are always limited to the diseases of known drug therapy, thus it is unsuitable for diseases without any existing drug therapy. Moreover, these methods give only qualitative information regarding the link between drugs and disease.

Drugs usually exert their therapeutic effect by binding to a specific protein or nucleic acid [15], the "one disease, one drug, one target" paradigm that has dominated the pharmaceutical industry for the past few decades. Clinically, however, many drugs bind "off-target" to proteins other than their primary targets, which can lead to unintended effects. In the primary disease group, this may lead to undesirable side effects but, in another disease group, these off-target effects may be beneficial and may thus represent a new indication for the drug [16]. Moreover, from a systems aspect, proteins do not always perform their functions in isolation but interact with other cellular components to form complexes or pathways [17]. Disease, as an intricate phenotype, is usually related to abnormalities in several pathways [18]. It is reasonable to infer that drugs producing therapeutic effects may do so not only by targeting the disease-associated protein(s) directly but also by modulating the pathways involved in the pathological process. With knowledge of drug targets and disease-associated proteins accumulating rapidly, it becomes possible to investigate the relationships between drugs and diseases at the pathway level. In this study, we propose a disease-oriented strategy for evaluating the drugdisease relationship using pathway profiling. To test this method, we took eight multi-target drugs (i.e. having more than three known targets) from the top 20 global best-selling drugs in 2010 (http://knol.google.com/k/krishan-maggon/topten-twenty-best-selling-drugs-2010/3fy5eowy8suq3/141\#).

\section{Data collection}

\subsection{Pathway selection}

A "pathway" is defined as a manually annotated basic biological functional unit. KEGG [19] serves as a comprehensive resource for pathway information. Considering that many basic pathways are involved in pathological disease processes, all of the disease pathways in KEGG are excluded. As a result, 185 basic pathways were collected, including
5703 genes.

\subsection{Disease- and disease-associated proteins}

In this work, we integrated two disease- and disease-associated protein databases, using (i) data from a text mining exercise to extract the relationship between $\mathrm{MeSH}$ items and genes [20] and (ii) collection of all disease-protein relationship pairs indicated as "curated" in the Comparative Toxicogenomics Database (CTD) [21]. We found 806 diseases with more than three disease-associated proteins, and 2616 proteins associated with disease.

\section{Method}

\subsection{Pathway profile construction}

In this work, a hypergeom test was taken to evaluate the enrichment of disease-associated proteins in each pathway. Every disease then obtained a vector with 185 dimensions with each element in the vector being a $p$-value of the hypergeom test. We define the vector as the pathway profile. The same approach was used for drug targets.

\subsection{Drug-disease association and significance level}

(i) Calculating drug-disease association. We assume that a drug may have a regulatory effect on a disease if both significantly influence the same pathways. Hence, a formula (1) was defined to evaluate the association of drug and disease. Pathways that contained two or more disease-associated proteins and two or more drug targets and that had enriched $p$-values less than 0.01 were identified as being significantly affected by both disease and drug,

$$
S_{\text {drug,disease } i}=-\lg \sum \sqrt{p_{\text {drug }, k} \times p_{\text {disease } i, k}} .
$$

Here, $p_{\text {drug, } k}$ is the enriched $p$-value of drug on pathway " $k$ "; $p_{\text {disease } i, k}$ is the enriched $p$-value of disease " $i$ " on pathway " $k$ "; $k$ is the pathway which is significantly affected by both drug and disease " $i$ ".

(ii) Random pathway profile construction of drug. The difference in the number of disease-associated proteins may lead to bias of the association score, making it necessary to evaluate the specificity of associations between drug and disease. While retaining the pathway profile sequence of the drug, random pathway profiles of the drug were generated by randomly sorting the pathway 100000 times. For each random profile, the $S_{\text {random,disease }}$ was calculated according to formula (1).

(iii) Evaluation of association specificity between drug and disease. In this study, the $Z$-score was used to evaluate the specificity of the association between the drug and disease $(Z>2.33)$, 


$$
Z_{\text {drug,disease } i}=\frac{S_{\text {drug,disease } i}-\operatorname{average}\left(S_{\text {random,disease } i}\right)}{\operatorname{std}\left(S_{\text {random,disease } i}\right)} .
$$

Here, average $\left(S_{\text {random, disease }}\right)$ is the average association score between random cases and disease $i, \operatorname{std}\left(S_{\text {random, disease }}\right)$ is the standard variation of association between random cases and disease $i$.

\section{Results}

Although most drugs are generally designed using the "one disease, one drug, one-target" paradigm, drugs with multiple targets nevertheless constitute an appreciable proportion of known drugs. In the top 20 global best-selling drugs of 2010, $13(65 \%)$ are multi-target drugs. Among these are eight drugs with more than three targets, namely Etanercept, Bevacizumab, Aripiprazole, Rituximab, Adalimumab, Quetiapine, Trastuzumab and Olanzapine. These drugs were used as test samples to predict potential therapeutic functions or side effects. According to their advertised pharmacological function, these drugs are focused on treating three main types of disease: psychosis, rheumatoid arthritis and cancer. For each of these disease areas, one drug is discussed in detail below. For the other five drugs, see Figures S1-S5.

\subsection{Etanercept}

Etanercept is a protein-based drug used in the treatment of autoimmune diseases for its actions as a TNF inhibitor. It is co-marketed by Amgen and Pfizer, with the FDA-approved therapeutic function of treating rheumatoid arthritis, juvenile idiopathic arthritis and ankylosing spondylitis. Besides TNF-alpha, etanercept has another 13 targets of unknown function described in DrugBank. Table 1 lists the top 10 diseases associated with Etanercept, as predicted by our method.

Table 1 Top 10 Etanercept-associated diseases based on pathway profile

\begin{tabular}{llll}
\hline MeSH ID & \multicolumn{1}{c}{ Disease } & \multicolumn{1}{c}{ Score } & \multicolumn{1}{c}{$Z$} \\
\hline D001172 $^{\text {a) }}$ & Arthritis, rheumatoid & 36.10333 & 7.030134 \\
D008180 $^{\text {b) }}$ & Lupus erythematosus, systemic & 35.463 & 8.174273 \\
D010518 $^{\text {b) }}$ & Periodontitis & 34.2678 & 8.566442 \\
D008216 & Lymphocytic choriomeningitis & 26.62368 & 6.239536 \\
D001943 & Breast neoplasms & 25.96673 & 2.991214 \\
D005909 $^{\text {b }}$ & Glioblastoma & 24.95259 & 3.182368 \\
D011565 & Psoriasis & 24.40725 & 4.754224 \\
D010022 & Osteopetrosis & 23.35165 & 6.728951 \\
D000026 & Abortion, habitual & 23.2841 & 6.153638 \\
D008288 & Malaria & 22.23864 & 9.206756 \\
\hline
\end{tabular}

a) Primary therapeutic function approved by FDA; b) supported by literature.
As shown in Table 1, the FDA-approved indication for this drug, rheumatoid arthritis, ranked most highly. Another five predicted diseases in which Etanercept could be applied have been validated by literature. Notably, psoriasis, for which Etanercept has undergone clinical phase III trials [22], is also included in this top 10. Furthermore, although literature reporting the use of Etanercept in periodontitis is limited, this, like rheumatoid arthritis, is a chronic inflammatory disease and shares several risk factors and pathological processes [23]. The prevalence of periodontal disease is known to increase two-fold among patients with rheumatoid arthritis compared to the general population [24], and Di Paola et al. [25] found that treatment with Etanercept reduces the development of the inflammatory and tissue injury events associated with periodontitis in a rat model.

Systemic lupus erythematosus is a systemic autoimmune disease that can affect any part of the body. Several studies have suggested that Etanercept can induce the pathological process of this disease [26-28]. TNF-alpha is also an important cytokine in the process of breast cancer development, and Madhusudan et al. [29] discovered, in phase II studies with 16 samples, that Etanercept could attenuate breast cancer without major adverse events.

Elevation of sTNF-R1 and sTNF-R2 is a marker of normal human pregnancy. In women who suffer habitual abortion, there is no elevation of sTNF-R1 and sTNF-R2 levels during pregnancy [30]. Treatment with inhibitors of tumor necrosis factor and intravenous immunoglobulin was found to improve live birth rates in women with habitual abortion [31].

No literature evidence was found for direct association between Etanercept and lymphocytic choriomeningitis, glioblastoma, osteoporosis or malaria. However, some indirect evidence may exist to infer associations. From the pathway profiles of Etanercept and lymphocytic choriomeningitis, Etanercept may regulate the disease through one or more of: the hematopoietic cell lineage; the NOD-like receptor signaling pathway; natural killer cell-mediated cytotoxicity; the adipocytokine signaling pathway; and/or osteoclast differentiation. For instance, Rodas et al. [32] found that circulating natural killer cell numbers decrease soon after infection of rhesus macaques with lymphocytic choriomeningitis virus. Etanercept is known to reduce the levels of natural killer cells [33], so may affect lymphocytic choriomeningitis by regulating natural killer cell-mediated cytotoxicity.

TNF-alpha induces angiogenic factor up-regulation in malignant glioma cells [34]. Etanercept may therefore modulate glioblastoma by inhibiting TNF-alpha. Perturbation of the balance between bone formation and resorption can result in osteopetrosis or osteoporosis [35] and TNF-alpha is a known factor in the pathogenesis of various forms of bone loss, as supported by both experimental and clinical evidence [36]. Considering the overlap of significantly affected pathways, osteoclast differentiation could be a pathway targeted by Etanercept to influence osteopetrosis. 
Finally, Depinay et al. [37,38] found that treatment with TNF-alpha prevents the development of human and rodent malarial pre-erythrocytic stages. Therefore, Etanercept may increase the risk of malarial infection through its inhibitory actions on TNF-alpha.

\subsection{Trastuzumab}

Trastuzumab is an anti-HER2 humanized monoclonal antibody that interferes with the HER2/neu receptor, and is approved for the treatment of ErbB-2-positive metastatic breast cancer. DrugBank contains 13 targets of trastuzumab, including the primary therapeutic target, ErbB-2. The association score of the pathway profile between trastuzumab and diseases was calculated according to the method described above. The top 10 predicted associations are listed in Table 2.

Besides breast neoplasms, four other cancers (ovarian, head-and-neck and stomach neoplasms, and glioblastoma) appear in the top 10. Several studies show that the ErbB signaling pathway, which includes the Trastuzumab target, ErbB2, is involved in the pathology of these cancers [38-41]. In the clinical pharmacological studies, Trastuzumab was found to inhibit the growth of tumor cells by affecting RhoB and PTEN in an ovarian carcinoma model without HER-2 amplification or overexpression [42]. Mineo et al. [43] reported the ability of Trastuzumab to induce apoptosis and cellular-dependent cytotoxicity in ErbB-2-expressing glioblastoma multiforme cell lines. In addition, Trastuzumab was shown to enhance the antitumor effect of Gefitinib on head and neck squamous cell carcinoma [44]. The ToGA trial, an international multicenter phase III clinical study involving 24 countries, showed that Trastuzumab is effective in prolonging survival in HER2-positive carcinoma of the stomach [45]. In cytology studies, HER-2 protein is overexpressed in $20 \%-66 \%$ of resected non-small cell lung cancer, and Trastuzumab was able to inhibit proliferation of such cancer cells [46-48].

Table 2 Top 10 Trastuzumab-associated diseases based on pathway profile

\begin{tabular}{llcc}
\hline MeSH ID & \multicolumn{1}{c}{ Disease } & Score & $Z$ \\
\hline D001943 $^{\text {a) }}$ & Breast neoplasms & 29.37911 & 3.454263 \\
D010051 $^{\text {b) }}$ & Ovarian neoplasms & 30.12039 & 3.567726 \\
D005909 $^{\text {b) }}$ & Glioblastoma & 27.86462 & 3.634425 \\
D013274 & Stomach neoplasms & 27.64782 & 4.358746 \\
D018805 & Sepsis & 25.68048 & 3.268015 \\
D006258 & Head and neck neoplasms & 24.57259 & 3.221091 \\
D008180 & Lupus erythematosus, systemic & 23.71224 & 5.058859 \\
D008175 & Lung neoplasms & 23.51886 & 2.42144 \\
D010518 & Periodontitis & 23.5185 & 5.506411 \\
D001172 & Arthritis, rheumatoid & 20.43737 & 3.528464 \\
\hline
\end{tabular}

a) Primary therapeutic function approved by FDA; b) supported by literature.
Troponin-I was discovered as prognostic mortality indicator in sepsis patients [49]. However, the expression level of troponin-I is elevated after treatment with Trastuzumab [50], suggesting a potential risk of sepsis during Trastuzumab therapy.

Rheumatoid arthritis is a chronic inflammatory disease, characterised by hyperplasia of synovial tissue and an abnormal immune response [51]. ErbB-2 is involved in rheumatoid synovial cell growth and Trastuzumab, as an inhibitor of ErbB-2, was able to inhibit this process. Inhibition of ErbB-2 may therefore provide a new approach to treating rheumatoid arthritis [52]. As with Etanercept, Trastuzumab may have a role in the therapy of systemic lupus erythematosus. The Fc-gamma receptor plays an important role in the treatment of SLE [53,54] and polymorphisms in this receptor show significant associations with periodontitis [55-57]. Because Trastuzumab has been shown to engage in the activation/inhibition of Fc-gamma [58], it may be able to modulate these two Fc-gamma-related diseases.

\subsection{Aripiprazole}

Aripiprazole is an atypical antipsychotic that acts as a dopamine D2 receptor partial agonist. In this study, 25 targets collected in DrugBank were used to generate the pathway profile. Table 3 lists the top 10 predictions.

The four diseases most closely associated with Aripiprazole are psychiatric disorders, with schizophrenia and bipolar disorder being the two diseases for which Aripiprazole is an approved treatment. Although not formally approved, Aripiprazole was reported to also have efficacy when applied in the therapy of autistic spectrum disorders [59-64]. In a small sample study, Aripiprazole efficiently reduced attention deficit hyperactivity disorder without producing any cognitive impairment [65].

Obesity, diabetes mellitus and epilepsy were also strongly associated side effects predicted by our model. The theory

Table 3 Top 10 Aripiprazole-associated diseases based on pathway profile

\begin{tabular}{clcc}
\hline MeSH ID & \multicolumn{1}{c}{ Disease } & Score & $Z$ \\
\hline D012559 & Schizophrenia & 30.68264 & 5.613725 \\
D001321 $^{\text {b) }}$ & Autistic disorder & 27.05887 & 4.973414 \\
D001714 $^{\text {a) }}$ & Bipolar disorder & 25.20179 & 6.095611 \\
D001289 $^{\text {b) }}$ & Attention deficit disorder with & 23.49487 & 8.090225 \\
D002534 & Hyperactivity & 23.4884 & 5.244597 \\
D009765 & Obesity & 22.86035 & 6.226287 \\
D004829 & Epilepsy, generalized & 21.77394 & 10.16691 \\
D004409 & Dyskinesia, drug-induced & 21.70412 & 9.777261 \\
D008881 & Migraine disorders & 21.26583 & 7.631553 \\
D003921 & Diabetes mellitus, experimental & 20.59053 & 6.530241 \\
\hline a) Primary & therapeutic function approved by FDA; b) supported by lit- \\
erature. & & &
\end{tabular}


that hypersensitivity of the dopaminergic system may be involved in the pathogenesis of migraine has been supported by various authors on the basis of clinical, pharmacological and recent genetic evidence $[66,67]$, and the D2 partial agonist, Aripiprazole, was found to offer relief from migraine headache [68]. Several studies reported that Aripiprazole might precipitate dyskinesia and other movement disorders [69-71]. Calcium signalling is always altered during hypoxia [72], and Aripiprazole is able to modulate calcium signaling by regulating the serotonin 5-HT2C receptor [73]. This may lead to an association between Aripiprazole and hypoxic brain damage.

\section{Conclusion and discussion}

The FDA-approved therapeutic functions of the eight test drugs are all included in the top 10 predicted results. Furthermore, $60 \%$ of the predictions were supported by clinical evidence and/or scientific literature, validating the accuracy of our methods. The unidentified functions may represent potential new indications for the drug in question. Our method is developed based on two biological hypotheses: firstly, that proteins always perform their functions within pathways, not in isolation; and secondly, that drugs exert therapeutic effects by regulating the pathways involved in disease pathology rather than directly targeting diseaseassociated proteins. By fully considering the known targets of the drug, prediction of pharmacological indications based on association between drugs and diseases seems reasonable. This unbiased, disease-oriented strategy could be used to predict new indications, without the constraint of the existing pharmacological functional space of the drugs.

Many pathways contribute to the pathological process of disease. Our method comprehensively models the relationships between drugs and diseases, rather than simply the primary target pathway, which we hope will lead to the discovery of novel therapeutic strategies. For example, disruption of the immune system is the most frequently used approach in the therapy of the autoimmune disorder, rheumatoid arthritis. However, Trastuzumab, a monoclonal antibody of ErbB-2, is an effective agent in treating rheumatoid arthritis, and does so via a pathway unrelated to immunological modulation. According to the literature, targeting growth factors is a promising approach for the treatment of rheumatoid arthritis because of the resultant inhibition of synovial tissue hyperplasia.

Multiple targets are necessary in building the pathway profile of a drug, rendering it unsuitable for drugs with only a single target. Nevertheless, by considering the association drugs and disease at the pathway level, it may be possible to predict new indications of existing drugs based on gene expression data. Disease-oriented and pathway-based approaches can be used to complement existing methods and may provide a new perspective in drug repositioning and the evaluation of side effects.

We thank Dr. Li Yong for providing us with the database of disease-and disease-associated proteins. This work was supported by the National Natural Science Foundation of China (30900832), Open Project Program Foundation of Key Laboratory of Liver and Kidney Diseases (Shanghai University of Traditional Chinese Medicine), Ministry of Education Program for New Century Excellent Talents in University (NCET-08-0399), "Shu Guang" Project by Shanghai Municipal Education Commission and Shanghai Education Development Foundation (07SG22), and grants from Ministry of Science and Technology China (2012ZX10005001).

1 Adams $\mathrm{C} \mathrm{P}$, Brantner V V. Estimating the cost of new drug development: Is it really 802 million dollars? Health Aff (Millwood), 2006, 25: 420-428

2 Krantz A. Protein-site targeting. Diversification of the drug discovery process. Nat Biotechnol, 1998, 16: 1294

3 Day M. The bitterest pill. Nurs Times, 1996, 92: 16-17

4 Kola I, Landis J. Can the pharmaceutical industry reduce attrition rates? Nat Rev Drug Discov, 2004, 3: 711-715

5 Calabrese L, Fleischer A B. Thalidomide: Current and potential clinical applications. Am J Med, 2000, 108: 487-495

6 Amato R J. Thalidomide: An antineoplastic agent. Curr Oncol Rep, 2002, 4: 56-62

7 Matthews S J, McCoy C. Thalidomide: A review of approved and investigational uses. Clin Ther, 2003, 25: 342-395

8 Gasparini G, Morabito A, Magnani E, et al. Thalidomide: An old sedative-hypnotic with anticancer activity? Curr Opin Investig Drugs, 2001, 2: 1302-1308

9 Ashburn $\mathrm{T}$ T, Thor $\mathrm{K}$ B. Drug repositioning: Identifying and developing new uses for existing drugs. Nat Rev Drug Discov, 2004, 3: 673-683

10 Chong C R, Sullivan D J Jr. New uses for old drugs. Nature, 2007, 448: 645-646

11 Keiser M J, Setola V, Irwin J J, et al. Predicting new molecular targets for known drugs. Nature, 2009, 462: 175-181

12 Campillos M, Kuhn M, Gavin A C, et al. Drug target identification using side-effect similarity. Science, 2008, 321: 263-266

13 Iorio F, Bosotti R, Scacheri E, et al. Discovery of drug mode of action and drug repositioning from transcriptional responses. Proc Natl Acad Sci USA, 2010, 107: 14621-14626

14 He L, Luo H, Chen J, et al. DRAR-CPI: A server for identifying drug repositioning potential and adverse drug reactions via the chemicalprotein interactome. Nucleic Acids Res, 2011, 39: W492-W498

15 Drews J. Drug discovery: A historical perspective. Science, 2000, 287: 1960-1964

16 Paolini G V, Shapland R H, van Hoorn W P, et al. Global mapping of pharmacological space. Nat Biotechnol, 2006, 24: 805-815

17 Barabasi A L, Oltvai Z N. Network biology: Understanding the cell's functional organization. Nat Rev Genet, 2004, 5: 101-113

18 Barabasi A L, Gulbahce N, Loscalzo J. Network medicine: A network-based approach to human disease. Nat Rev Genet, 2011, 12: $56-68$

19 Kanehisa M, Goto S, Furumichi M, et al. KEGG for representation and analysis of molecular networks involving diseases and drugs. Nucleic Acids Res, 2010, 38: D355-360

20 Li Y, Agarwal P. A pathway-based view of human diseases and disease relationships. PLoS ONE, 2009, 4: e4346

21 Davis A P, King B L, Mockus S, et al. The comparative toxicogenomics database: Update 2011. Nucleic Acids Res, 2011, 39: D10671072

22 Feldman S R, Kimball A B, Krueger G G, et al. Etanercept improves the health-related quality of life of patients with psoriasis: Results of a phase III randomized clinical trial. J Am Acad Dermatol, 2005, 53: 887-889

23 De Smit M J, Brouwer E, Vissink A, et al. Rheumatoid arthritis and 
periodontitis: A possible link via citrullination. Anaerobe, 2011, 17: 196-200

24 Berthelot J M, Le Goff B. Rheumatoid arthritis and periodontal disease. Joint Bone Spine, 2010, 77: 537-541

25 Di Paola R, Mazzon E, Muia C, et al. Effects of etanercept, a tumour necrosis factor-alpha antagonist, in an experimental model of periodontitis in rats. Br J Pharmacol, 2007, 150: 286-297

26 Swale V J, Perrett C M, Denton C P, et al. Etanercept-induced systemic lupus erythematosus. Clin Exp Dermatol, 2003, 28: 604607

27 Kang M J, Lee Y H, Lee J. Etanercept-induced systemic lupus erythematosus in a patient with rheumatoid arthritis. J Korean Med Sci, 2006, 21: 946-949

28 Shakoor N, Michalska M, Harris C A, et al. Drug-induced systemic lupus erythematosus associated with etanercept therapy. Lancet, 2002, 359: 579-580

29 Madhusudan S, Foster M, Muthuramalingam S R, et al. A phase II study of etanercept (Enbrel), a tumor necrosis factor alpha inhibitor in patients with metastatic breast cancer. Clin Cancer Res, 2004, 10: 6528-6534

30 Chernyshov V P, Vodyanik M A, Pisareva S P. Lack of soluble TNF-receptors in women with recurrent spontaneous abortion and possibility for its correction. Am J Reprod Immunol, 2005, 54: 284-291

31 Winger E E, Reed J L. Treatment with tumor necrosis factor inhibitors and intravenous immunoglobulin improves live birth rates in women with recurrent spontaneous abortion. Am J Reprod Immunol, 2008, 60: 8-16

32 Rodas J D, Cairo C, Djavani M, et al. Circulating natural killer and gammadelta $\mathrm{T}$ cells decrease soon after infection of rhesus macaques with lymphocytic choriomeningitis virus. Mem Inst Oswaldo Cruz, 2009, 104: 583-591

33 Wallace D J. The use of etanercept and other tumor necrosis factor-alpha blockers in infertility: It's time to get serious. J Rheumatol, 2003, 30: 1897-1899

34 Nabors L B, Suswam E, Huang Y, et al. Tumor necrosis factor alpha induces angiogenic factor up-regulation in malignant glioma cells: A role for RNA stabilization and HuR. Cancer Res, 2003, 63: 4181-4187

35 Lazner F, Gowen M, Pavasovic D, et al. Osteopetrosis and osteoporosis: Two sides of the same coin. Hum Mol Genet, 1999, 8: 1839-1846

36 Boyce B F, Li P, Yao Z, et al. TNF-alpha and pathologic bone resorption. Keio J Med, 2005, 54: 127-131

37 Depinay N, Franetich J F, Gruner A C, et al. Inhibitory effect of TNF-alpha on malaria pre-erythrocytic stage development: Influence of host hepatocyte/parasite combinations. PLoS ONE, 2011, 6: e17464

38 Maihle N J, Baron A T, Barrette B A, et al. EGF/ErbB receptor family in ovarian cancer. Cancer Treat Res, 2002, 107: 247-258

39 Andersson U, Schwartzbaum J, Wiklund F, et al. A comprehensive study of the association between the EGFR and ERBB2 genes and glioma risk. Acta Oncol, 2010, 49: 767-775

40 Ratushny V, Astsaturov I, Burtness B A, et al. Targeting EGFR resistance networks in head and neck cancer. Cell Signal, 2009, 21: 1255-1268

41 Begnami M D, Fukuda E, Fregnani J H, et al. HER2 and HER3 are Predictors of poor outcome. J Clin Oncol, 2011, 29: 3030-3036

42 Delord J P, Quideau S, Rochaix P, et al. Trastuzumab induced in vivo tissue remodelling associated in vitro with inhibition of the active forms of AKT and PTEN and RhoB induction in an ovarian carcinoma model. Br J Cancer, 2010, 103: 61-72

43 Mineo J F, Bordron A, Quintin-Roue I, et al. Recombinant humanised anti-HER2/neu antibody (Herceptin) induces cellular death of glioblastomas. Br J Cancer, 2004, 91: 1195-1199

44 Kondo N, Ishiguro Y, Kimura M, et al. Antitumor effect of gefitinib on head and neck squamous cell carcinoma enhanced by trastuzumab. Oncol Rep, 2008, 20: 373-378

45 Albarello L, Pecciarini L, Doglioni C. HER2 testing in gastric cancer. Adv Anat Pathol, 2011, 18: 53-59
46 Azzoli C G, Krug L M, Miller V A, et al. Trastuzumab in the treatment of non-small cell lung cancer. Semin Oncol, 2002, 29: 59-65

47 Langer C J, Stephenson P, Thor A, et al. Trastuzumab in the treatment of advanced non-small-cell lung cancer: Is there a role? Focus on Eastern Cooperative Oncology Group study 2598. J Clin Oncol, 2004, 22: 1180-1187

48 Ferrone M, Motl S E. Trastuzumab for the treatment of non-smallcell lung cancer. Ann Pharmacother, 2003, 37: 1904-1908

49 John J, Woodward D B, Wang Y, et al. Troponin-I as a prognosticator of mortality in severe sepsis patients. J Crit Care, 2010, 25: 270-275

50 Telli M L, Witteles R M. Trastuzumab-related cardiac dysfunction. J Natl Compr Canc Netw, 2011, 9: 243-249

51 Gay S, Gay R E, Koopman W J. Molecular and cellular mechanisms of joint destruction in rheumatoid arthritis: Two cellular mechanisms explain joint destruction? Ann Rheum Dis, 1993, 52: S39-S47

52 Satoh K, Kikuchi S, Sekimata M, et al. Involvement of ErbB-2 in rheumatoid synovial cell growth. Arthritis Rheum, 2001, 44: 260265

53 Manger K, Repp R, Jansen M, et al. Fcgamma receptor IIa, IIIa, and IIIb polymorphisms in German patients with systemic lupus erythematosus: Association with clinical symptoms. Ann Rheum Dis, 2002, 61: 786-792

54 Brambila-Tapia A J, Davalos-Rodriguez I P. Fcgamma receptor polymorphisms and systemic lupus erythematosus. Rev Invest Clin, 2009, 61: 66-72

55 Wolf D L, Neiderud A M, Hinckley K, et al. Fcgamma receptor polymorphisms and periodontal status: A prospective follow-up study. J Clin Periodontol, 2006, 33: 691-698

56 Yamamoto K, Kobayashi T, Grossi S, et al. Association of Fcgamma receptor IIa genotype with chronic periodontitis in Caucasians. J Periodontol, 2004, 75: 517-522

57 Loos B G, Leppers-Van de Straat F G, Van de Winkel J G, et al. Fcgamma receptor polymorphisms in relation to periodontitis. J Clin Periodontol, 2003, 30: 595-602

58 Musolino A, Naldi N, Bortesi B, et al. Immunoglobulin G fragment C receptor polymorphisms and clinical efficacy of trastuzumab-based therapy in patients with HER-2/neu-positive metastatic breast cancer. J Clin Oncol, 2008, 26: 1789-1796

59 Erickson C A, Stigler K A, Posey D J, et al. Aripiprazole in autism spectrum disorders and fragile $\mathrm{X}$ syndrome. Neurotherapeutics, 2010, 7: 258-263

60 Pardini M, Guida S, Gialloreti L E. Aripiprazole treatment for coprophagia in autistic disorder. J Neuropsychiatry Clin Neurosci, 2010, 22: e433-451

61 Blankenship K, Erickson C A, Stigler K A, et al. Aripiprazole for irritability associated with autistic disorder in children and adolescents aged 6-17 years. Ped Health, 2010, 4: 375-381

62 Marcus R N, Owen R, Manos G, et al. Aripiprazole in the treatment of irritability in pediatric patients (aged 6-17 years) with autistic disorder: results from a 52-week, open-label study. J Child Adolesc Psychopharmacol, 2011, 21: 229-236

63 Owen R, Sikich L, Marcus R N, et al. Aripiprazole in the treatment of irritability in children and adolescents with autistic disorder. Pediatrics, 2009, 124: 1533-1540

64 Robb A S, Andersson C, Bellocchio E E, et al. Safety and tolerability of aripiprazole in the treatment of irritability associated with autistic disorder in pediatric subjects (6-17 years old): Results from a pooled analysis of 2 studies. Prim Care Companion CNS Disord, 2011, 13: PCC. $10 \mathrm{~m} 01008$

65 Findling R L, Short E J, Leskovec T, et al. Aripiprazole in children with attention-deficit/hyperactivity disorder. J Child Adol Psychopharmacol, 2008, 18: 347-354

66 Del Zompo M. Dopaminergic hypersensitivity in migraine: Clinical and genetic evidence. Funct Neurol, 2000, 15: 163-170

67 Cubo E, Kompoliti K, Leurgans S E, et al. Dopaminergic hypersensitivity in patients with Parkinson disease and migraine. Clin Neuropharmacol, 2004, 27: 30-32

68 LaPorta L D. Relief from migraine headache with aripiprazole 
treatment. Headache, 2007, 47: 922-926

69 Maytal G, Ostacher M, Stern T A. Aripiprazole-related tardive dyskinesia. CNS Spectr, 2006, 11: 435-439

70 Abbasian C, Power P. A case of aripiprazole and tardive dyskinesia. J Psychopharmacol, 2009, 23: 214-215

71 Pena M S, Yaltho T C, Jankovic J. Tardive dyskinesia and other movement disorders secondary to aripiprazole. Mov Disord, 2011, 26:
$147-152$

72 Kanatous S B, Mammen P P A, Rosenberg P B, et al. Hypoxia reprograms calcium signaling and regulates myoglobin expression. Cell Physiol, 2009, 296: C393-C402

73 Zhang J Y, Kowal D M, Nawoschik S P, et al. Distinct functional profiles of aripiprazole and olanzapine at RNA edited human 5-HT2C receptor isoforms. Biochem Pharmacol, 2006, 71: 521-529

Open Access This article is distributed under the terms of the Creative Commons Attribution License which permits any use, distribution, and reproduction in any medium, provided the original author(s) and source are credited.

\section{Supporting Information}

Table S1 Top 10 Bevacizumab-associated diseases based on pathway profile

Table S2 Top 10 Adalimumab-associated diseases based on pathway profile

Table S3 Top 10 Rituximab-associated diseases based on pathway profile

Table S4 Top 10 Quetiapine-associated diseases based on pathway profile

Table S5 Top 10 Olanzapine-associated diseases based on pathway profile

The supporting information is available online at csb.scichina.com and www.springerlink.com. The supporting materials are published as submitted, without typesetting or editing. The responsibility for scientific accuracy and content remains entirely with the authors. 